\title{
A PAIRWISE APPROXIMATION IN THE HUBBARD MODEL
}

\author{
J. SzEFTEL \\ C.E. Saclay, LLB (CEA-CNRS)/DRECAM, 91191, Gif-sur-Yvette, Cedex, France
}

The Hubbard Hamiltonian is projected onto a representation consisting of electron pairs characterised by the momentum of their centre of mass. Within this approximation the electron gas can be viewed as a collection of subsets, each of which contains a constant number of electron pairs, all having the same centre of mass momentum. As these subsets are decoupled, the Hubbard Hamiltonian is diagonalised to give two types of many-body eigenstates: correlated and uncorrelated. The uncorrelated pairs build up an ideal Fermi gas. Excellent agreement is found for the uncorrelated energy calculated at zero temperature in one dimension between this model and the Bethe ansatz, for arbitrary electron concentration and magnitude of the electron interaction. The correlated states turn out to be of the BCS type. PACS numbers: 71.10.+x, 71.45.-d, 74.20.-z

The Hubbard model has long been recognised as a paradigm in the manybody problem due to its relevance to magnetism and superconductivity $[1,2]$. Its characteristic feature is a two-body force, instrumental only for two electrons scattering at the same site. Its properties are believed to be different according to whether the Hubbard coupling measured by $U$ is attractive $(U<0)$ or repulsive $(U>0)$. For $U<0$ at low temperature $T$, the electrons are supposed to condense in a superconducting state according to the BCS picture [3]. At $U>0$ except for a paucity of exact results [4], there is no general agreement since the different approximations $[5,6]$ yield contradictory results: the electron gas is claimed to be a Fermi liquid of the Landau- or Luttinger type [7-9], ferromagnetic or antiferromagnetic $[1,5]$.

However, the above mentioned analyses share a common feature: the manybody wave functions are all defined in the same Hilbert space $S_{1}$ of Slater determinants, each of which contains the same number of electrons as given by concentration. The purpose of this work is to introduce another Hilbert space, then to project the Hubbard Hamiltonian onto this space and to investigate the properties of the associated eigenstates.

A crystal containing $n$ sites $(n \gg 1)$ and $2 n_{x}$ electrons is considered. The electron concentration is given by $x=2 n_{x} / n$. As the electrons are distributed in a single band, the Pauli principle requires: $0 \leq x \leq 2$. The Hubbard Hamiltonian reads in $S_{1}$ :

$$
h=\sum_{k, \zeta} E(k) c_{k, \zeta}^{+} c_{k, \zeta}+\frac{U}{n} \sum_{K, k, k^{\prime}} c_{k, \zeta}^{+} c_{K-k,-\zeta}^{+} c_{k^{\prime},-\zeta} c_{K-k^{\prime}, \zeta}
$$


where $c_{k, \zeta}^{(+)}$destroys (creates) an electron of wave number $k$ and spin $\zeta= \pm 1 / 2$, and the sums with respect to $K, k, k^{\prime}$ are performed in the Brillouin zone (BZ). The one-electron dispersion is written

$$
E(k)=D \sum_{\alpha=1}^{d} \cos k_{\alpha}
$$

where $k_{\alpha=1, \ldots d}$ are the components of $k$ for a crystal dimension $d=1,2$ or 3 and the bandwidth is proportional to $D$. Following BCS $[3,10]$, the electron pair operators are introduced

$$
b_{ \pm 1}^{(+)}\left(k, k^{\prime}\right)=c_{k, \pm \zeta}^{(+)} c_{k^{\prime}, \pm \zeta}^{(+)}, \quad b_{0}^{(+)}\left(k, k^{\prime}\right)=c_{k, \zeta}^{(+)} c_{k^{\prime},-\zeta}^{(+)}
$$

where the order of $c_{k, \zeta}$ is reverted in the product giving $b_{\sigma=0, \pm 1}\left(k, k^{\prime}\right)$. Note that $b_{ \pm 1}^{(+)}\left(k, k^{\prime}\right)=b_{ \pm 1}^{(+)}\left(k^{\prime}, k\right)$ within the sign whereas $b_{0}^{(+)}\left(k, k^{\prime}\right) \neq b_{0}^{(+)}\left(k^{\prime}, k\right)$. Hence there are twice more $(\sigma=0)$ singlet pairs than $(\sigma=1)$ or $(\sigma=-1)$ triplet pairs. Because the operators $b_{\sigma}^{(+)}\left(k, k^{\prime}\right)$ have [10] commutation properties pertaining to both fermions and bosons, they are referred to as hard-core bosons [11]. Likewise the pair occupation number in any state $\Phi$ or $S_{1}$ containing an even number of electrons $\left(=\left\langle\Phi\left|b_{\sigma}^{+}\left(k, k^{\prime}\right) b_{\sigma}\left(k, k^{\prime}\right)\right| \Phi\right\rangle\right)$ equals 0 or 1 as for fermions. The Hubbard Hamiltonian in terms of these pair operators reads

$$
\begin{aligned}
h= & \sum_{k, K, \sigma=0, \pm 1}(E(k)+E(K-k)) b_{\sigma}^{+}(k, K-k) b_{\sigma}(k, K-k) \\
& +\frac{U}{n} \sum_{k, k^{\prime}, K} b_{0}^{+}(k, K-k) b_{0}\left(k^{\prime}, K-k^{\prime}\right),
\end{aligned}
$$

where the matrix elements of the one- and two-body parts $((E(k)+E(K-k))$ and $(U / n)$ of $h$ in Eq. (1) are calculated as $\left\langle 0\left|b_{\sigma}(k, K-k) h b_{\sigma}^{+}(k, K-k)\right| 0\right\rangle$ and $\left\langle 0\left|b_{0}(k, K-k) h b_{0}^{+}\left(k^{\prime}, K-k^{\prime}\right)\right| 0\right\rangle$ respectively $(|0\rangle$ denotes the no electron state). The expressions of $h$ in Eq. (1) and Eq. (4) are equivalent provided the electron number $\left(=2 n_{x}\right)$ is even. The Hamiltonian in Eq. (4) is recast as function of partial Hamiltonians $h_{\sigma=0, \pm 1}(K)$ :

$$
\begin{aligned}
& h_{\sigma}(K)=\sum_{k}(E(k)+E(K-k)) b_{\sigma}^{+}(k, K-k) b_{\sigma}(k, K-k) \\
& \quad+\frac{\delta U}{n} \sum_{k, k^{\prime}} b_{0}^{+}(k, K-k) b_{0}\left(k^{\prime}, K-k^{\prime}\right), \\
& h=\sum_{K, \sigma}^{k} h_{\sigma}(K),
\end{aligned}
$$

where $\delta=1$ for $\sigma=0$ and $\delta$ vanishes for $\sigma= \pm 1$.

The Hilbert space underlying this work $S_{2}$ is defined by giving the expression of a vector $\Phi$ of its basis

$$
\Phi=\prod_{K, \sigma} \otimes \Phi_{\sigma}(K)
$$


where the tensor product of $\Phi_{\sigma}(K)$ is done for $K$ running through the $\mathrm{BZ}$ and $\sigma=0, \pm 1$. Every $\Phi_{\sigma}(K)$ is a Slater determinant comprising $n_{\sigma}(K)$ of $(K, \sigma)$ pairs as defined in Eq. (3) $\left(k+k^{\prime}=K\right)$ :

$$
\Phi_{\sigma}(K)=\prod_{j=1}^{n_{\sigma}(K)} b_{\sigma}^{+}\left(k_{j}, K-k_{j}\right)|0\rangle .
$$

The number of electrons in $\Phi$ fulfils

$$
\sum_{K, \sigma} \frac{n_{\sigma}(K)}{n}=\frac{x}{2}
$$

The dimensions of $S_{1}$ and $S_{2}$ equal respectively

$$
\left.d_{1}=\left(\begin{array}{c}
2 n_{x} \\
2 n
\end{array}\right) \text { and } d_{2}=\left(\begin{array}{c}
n_{x} \\
2 \\
2 n
\end{array}\right)\right) .
$$

As a given $c_{k, \zeta}^{+}$can form a pair with any other $c_{k^{\prime}, \zeta^{\prime}}^{+}$and then goes into every $\Phi_{\sigma}(K)$ constituting $\Phi$, by contrast with a Slater determinant which vanishes if any $c_{k, \zeta}^{+}$comes in more than once, $d_{1}<d_{2}$ for $n_{x}>1$. Two many-body state $\Phi$ and $\Phi^{\prime}$ of $S_{2}$ corresponding to Slater determinants of $S_{1}$ are considered: $\Phi=\sum_{i=1}^{m} \Phi_{i}$ and $\Phi^{\prime}=\sum_{i=1}^{m} \Phi_{i}^{\prime}$ where $\Phi_{i}$ and $\Phi_{i}^{\prime}$ represent all equivalent expressions of the two Slater determinants reordered as given by Eq. (6). Hence $m=\left(\begin{array}{c}2 \\ 2 n_{x}\end{array}\right)$. It comes out

$$
\left\langle\Phi|h| \Phi^{\prime}\right\rangle=\sum_{i, j=1}^{m}\left\langle\Phi_{i}|h| \Phi_{j}^{\prime}\right\rangle .
$$

Every term on the right hand side of Eq. (9) is non-vanishing only if $\Phi_{i}$ and $\Phi_{j}^{\prime}$ differ from each other by at most one pair, in which case the matrix element on the left hand side of Eq. (8) has the same value in $S_{1}$ and $S_{2}$. Therefore $S_{2}$ contains $S_{1}$. However, since $\left\langle\Phi_{\sigma}(K)\left|c_{k, \zeta}^{+} c_{k, \zeta}\right| \Phi_{\sigma}(K)\right\rangle$, the occupation number of any $c_{k, \zeta}^{+}|0\rangle$ in $\Phi_{\sigma}(K)$ cannot exceed one thanks to $\Phi_{\sigma}(K)$ being a determinant, and because of Eq. (8), the Pauli principle requires in $S_{2}: 0 \leq\left\langle\Phi\left|c_{k, \zeta}^{+} c_{k, \zeta}\right| \Phi\right\rangle \leq 2$. This results from the fact that any $c_{k, \zeta}^{+}|0\rangle$ can at most come into $n$ of $\Phi_{0}(K)$ and $n$ of $\Phi_{ \pm 1}(K)$ simultaneously.

Allowing $h$ to act on $\Phi$ yields

$$
h \Phi=\sum_{K, \sigma}\left(h \Phi_{\sigma}(K)\right) \otimes\left(\prod_{\left(K^{\prime}, \sigma^{\prime}\right) \neq(K, \sigma)} \otimes \Phi_{\sigma^{\prime}}\left(K^{\prime}\right)\right)
$$

where $h \Phi_{\sigma}(K)$ is recast into (see Eq. (5)):

$$
h \Phi_{\sigma}(K)=h_{\sigma}(K) \Phi_{\sigma}(K)+\sum_{\left(K^{\prime}, \sigma^{\prime}\right) \neq(K, \sigma)} h_{\sigma^{\prime}}\left(K^{\prime}\right) \Phi_{\sigma}(K) .
$$

Every term $h_{\sigma^{\prime}}\left(K^{\prime}\right) \Phi_{\sigma}(K)$ produces Slater determinants comprising $n_{\sigma}(K)$ of pairs, all of which do not have the same $(K, \sigma)$. The basic approximation of this work consists of neglecting such terms, which entails

$$
h \Phi=\sum_{K, \sigma}\left(h_{\sigma}(K) \Phi_{\sigma}(K)\right) \otimes\left(\prod_{\left(K^{\prime}, \sigma^{\prime}\right) \neq(K, \sigma)} \otimes \Phi_{\sigma^{\prime}}\left(K^{\prime}\right)\right) .
$$


Consequently the task to find out the eigenstates of $h$ in $S_{2}$ amounts to diagonalising separately each $h_{\sigma}(K)$ :

$$
\begin{aligned}
& \left(h-\varepsilon_{\Psi}\right) \Psi=0 \Leftrightarrow\left(h_{\sigma}(K)-\varepsilon_{\sigma}(K)\right) \Psi_{\sigma}(K)=0, \\
& \Psi=\prod_{K, \sigma} \otimes \Psi_{\sigma}(K), \quad \varepsilon_{\Psi}=\prod_{K, \sigma} \varepsilon_{\sigma}(K) .
\end{aligned}
$$

The pair number operators $N_{\sigma=0, \pm 1}(K)$ are now introduced

$$
N_{\sigma}(K)=\sum_{k} b_{\sigma}^{+}(k, K-k) b_{\sigma}(k, K-k) .
$$

The following conservation rule holds:

$$
\left[h_{\sigma}(K), N_{\sigma}(K)\right]=0 \text {, }
$$

whence $\Psi_{\sigma}(K)$ is seen to be sought as a linear combination of $\Phi_{\sigma}(K)$ in $E q .(7)$ all having the same number of pairs $n_{\sigma}(K)$.

Noteworthy are two remarks:

i) BCS $[3,10]$ truncated $h$ in keeping only $h_{0}(K=0)$ and then looked for a variational eigenstate of $h_{0}(K=0)$, consisting in the $(K=0, \sigma=0)$ pairs. This work attempts to extend the BCS approach by taking into account all $(K, \sigma)$ simultaneously;

ii) Eq. (12) means that the $(K, \sigma)$ pair subsets are decoupled. In the approximation used here, the Hubbard scattering cannot cause an electron of the $(K, \sigma)$ subset to jump to any other $\left(K^{\prime}, \sigma^{\prime}\right)$ one. Nevertheless, inside any $(K, \sigma)$ subset, the electrons are described in terms of Slater determinants, whereby they behave like usual indistinguishable fermions. As the various $h_{\sigma}(K)$ in Eq. (5) are decoupled in terms of $b_{\sigma}^{(+)}(k, K-k)$, this procedure would be exact if the pairs could be regarded as distinct objects.

Equation (13) will be solved for triplet $(\sigma= \pm 1)$ pairs first. The index $\sigma= \pm 1$ will be denoted by $t$. The partial Hamiltonian $h_{t}(K)$ reads (see Eq. (5)):

$$
h_{t}(K)=\sum_{k}(E(k)+E(K-k)) b_{t}^{+}(k, K-k) b_{t}(k, K-k) .
$$

As $h_{t}(K)$ is diagonal in the pair basis $b_{t}^{+}(k, K-k)|0\rangle$, the eigenvector $\Psi_{t}(K)$ and eigenvalue $\varepsilon_{t}(K)$ read

$$
\Psi_{t}(K)=\prod_{i=1}^{n_{t}(K)} b_{t}^{+}\left(k_{i}, K-k_{i}\right)|0\rangle, \quad \varepsilon_{t}(K)=\prod_{i=1}^{n_{t}(K)}\left(E\left(k_{i}\right)+E\left(K-k_{i}\right)\right) .
$$

Since $\varepsilon_{t}(K)$ reduces to the sum of one-particle energy $E\left(k_{i}\right), \Psi_{t}(K)$ is referred to as an uncorrelated many-body eigenstate and the electrons involved in such uncorrelated pairs are realized to make up a gas of independent fermions, governed by the one-particle Hamiltonian $h_{t}$ :

$$
h_{t}=\sum_{k} E(k) c_{k, \zeta}^{+} c_{k, \zeta}
$$

It must be noticed that the Fermi-Dirac statistical behaviour is generally worked out $[7,8]$ under the only assumption that the allowed value of $\left\langle\Phi_{\sigma}(K)\left|c_{k, \zeta}^{+} c_{k, \zeta}\right| \Phi_{\sigma}(K)\right\rangle$ is either 0 or 1 , irrespective of the nature of the resulting many-body state $\Phi$ which 
may belong to $S_{1}, S_{2}$ or even another kind of Hilbert space. Hence at $T=0$, the concentration $x_{t}$ and energy $\varepsilon_{t}$ per site for triplet pairs read

$$
x_{t}=\int_{-d|D|}^{\mu} \rho(E) \mathrm{d} E, \quad \varepsilon_{t}=\int_{-d|D|}^{\mu} E \rho(E) \mathrm{d} E,
$$

where $\rho(E)$ is the one-particle density of states associated with the dispersion law of Eq. (2), normalised by $\int_{-d|D|}^{d|D|} \rho(E) \mathrm{d} E=1$, and the chemical potential $\mu$ is equal to the single electron Fermi energy.

The partial Schrödinger equation for singlet $(\sigma=0)$ pairs reads (see Eq. (5)):

$$
\begin{aligned}
& \left(\varepsilon_{0}(K)-h_{0}(K)\right) \Psi_{0}(K)=0, \\
& h_{0}(K)=\sum_{k}(E(k)+E(K-k)) b_{0}^{+}(k, K-k) b_{0}(k, K-k) \\
& \quad+\frac{U}{n} \sum_{k, k^{\prime}} b_{0}^{+}(k, K-k) b_{0}\left(k^{\prime}, K-k^{\prime}\right) .
\end{aligned}
$$

A mean-field assumption is made

$$
\left\langle\Psi_{0}(K)\left|b_{0}^{+}(k, K-k) b_{0}\left(k^{\prime}, K-k^{\prime}\right)\right| \Psi_{0}(K)\right\rangle=\sqrt{n_{k}\left(1-n_{k}\right) n_{k^{\prime}}\left(1-n_{k^{\prime}}\right)},
$$
where $n_{k}=\left\langle\Psi_{0}(K)\left|b_{0}^{+}(k, K-k) b_{0}(k, K-k)\right| \Psi_{0}(K)\right\rangle$. As $\Psi_{0}(K)$ is given by a linear combination of Slater determinants $\Psi_{0}(K)$ containing $n_{0}(K)$ of pairs, every $n_{k}$ lies between 0 and 1 . The $n_{k}$ fulfil

$$
\sum_{k} \frac{n_{k}}{n}=\frac{n_{0}(K)}{n}=\frac{x_{0}(K)}{2},
$$

where $x_{0}(K)$ is the electron concentration in $\Psi_{0}(K)$. The energy per site $\varepsilon_{0}(K)$ is then written

$$
\varepsilon_{0}(K)=\sum_{k}(E(k)+E(K-k)) \frac{n_{k}}{n}+U \Delta^{2}, \quad \Delta=\sum_{k} \frac{\sqrt{n_{k}\left(1-n_{k}\right)}}{n} .
$$

Expressing that $\partial \varepsilon_{0}(K) / \partial n_{k}=0$ under the constraint of Eq. (22) for any eigenstate $\Psi_{0}(K)$ leads for $n_{k}$ to the BCS expression [3,10,11]. Accordingly, $\varepsilon_{0}(K)$ is ascribed to the $(\Delta \neq 0)$ BCS value, given $E(k), U$ and $x_{0}(K)$. Since $\varepsilon_{0}(K)$ contains a contribution $U \Delta^{2}$, the corresponding many-body wave function is said to be correlated. However, this work is chiefly concerned with the $\Delta=0$ solution of Eqs. (23), which implies $n_{k}=0$ or 1 for every $k$ in the Brillouin zone (BZ) consistently with Eq. (21). Thus $\varepsilon_{0}(K)$ in Eq. (23) reduces to a sum of one-particle energies. Hence the $\Delta=0$ singlet pairs build up a Fermi gas of uncorrelated electrons obeying the one-particle Hamiltonian $h_{s}$ :

$$
h_{s}=\sum_{k, \zeta} E(k) c_{k, \zeta}^{+} c_{k, \zeta}+\frac{U}{n} \sum_{k, k^{\prime}} c_{k, \zeta}^{+} c_{k, \zeta} c_{k^{\prime},-\zeta}^{+} c_{k^{\prime},-\zeta} .
$$

$h_{s}$ is deduced from $\left\langle\Psi_{0}(K)|h| \Psi_{0}(K)\right\rangle$ by taking into account $\left\langle\Psi_{0}(K)\left|b_{0}^{+}(k, K-k) b_{0}\left(k^{\prime}, K-k\right)\right| \Psi_{0}(K)\right\rangle=0$ for $k \neq k^{\prime}$ in the uncorrelated $(\Delta=0)$ solution. The Hamiltonian $h_{s}$ is realized to be diagonal in the one-particle basis $c_{k, \zeta}^{+}|0\rangle$ with the dispersion law

$$
E^{\prime}(k, \pm \zeta)=E(k)+\frac{U x_{\mp \zeta}}{2}
$$


where $x_{ \pm \zeta}$ is the concentration of electrons with spin $\pm \zeta$ and the factor $1 / 2$ avoids double counting. The corresponding energies $\varepsilon_{ \pm \zeta}$ and concentrations $x_{ \pm \zeta}$ are given at $T=0$ by

$$
x_{ \pm \zeta}=\int_{-d|D|}^{\mu-\frac{U}{2} x_{\mp \zeta}} \rho(E) \mathrm{d} E, \quad \varepsilon_{ \pm \zeta}=\int_{-d|D|}^{\mu-\frac{U}{2} x_{\mp \zeta}} E \rho(E) \mathrm{d} E+\frac{U}{2} x_{\zeta} x_{-\zeta}
$$

where the one-particle density of states $\rho(E)$ of Eq. (19) has been halved to account for the singlet uncorrelated pairs accommodating at most $1 / 2$ electron per spin direction.

The ground state of a gas of independent electrons is in general non-degenerate and consists of the Fermi sea defined in $S_{1}$ by filling every one-particle state up to $\mu$ and leaving empty the states above. In $S_{2}$ there are three different ground states $\Phi_{t}, \Phi_{s}$ and $\Phi_{t} \otimes \Phi_{s}$ according to whether the uncorrelated electrons are accommodated in the triplet or singlet pairs exclusively or in both simultaneously. The energy of $\Phi_{t}$ and $\Phi_{s}$ are those of a Fermi gas of dispersion law given by Eq. (2) and (25) respectively. That of $\Phi_{t} \otimes \Phi_{s}$ is worked out by solving Eqs. (19) and (26). This yields the energy $\varepsilon_{u}\left(=\varepsilon_{t}+\varepsilon_{\zeta}+\varepsilon_{-\zeta}\right)$ of uncorrelated singlet and triplet pairs per site versus concentration $x\left(=x_{t}+x_{\zeta}+x_{-\zeta}\right)$ at $T=0$. There are two types of solution: paramagnetic $\left(x_{\zeta}=x_{-\zeta}\right)$ and magnetic $\left(x_{\zeta} \neq x_{-\zeta}\right)$. The $d=1$ paramagnetic solution will be now compared with the Bethe wave-function based result [12] because the latter is also typical of independent particles distributed according to a density of states renormalised by the Hubbard coupling [4]. The comparison is illustrated in Fig. 1. The results of this work are found to be lower than those of Ref. [12] (except for $U=0$ and $\infty$ wherein both sets coincide because the two-body Hubbard interaction vanishes). This is not astonishing since

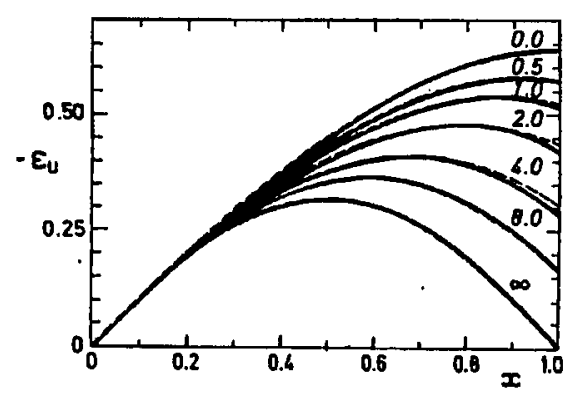

Fig. 1. Plot of the ground-state energy $\varepsilon_{\mathfrak{u}}$ of uncorrelated electrons in the $d=1$ Hubbard model at $T=0$ versus electron concentration $x$. The results of this work and of Ref. [12] are pictured in dashed and solid lines, respectively. The energy unit is $|D|$. Each curve is labelled with the corresponding value of $U$. 
the ground state of this work has been searched in a Hilbert space $S_{2}$ which is larger than $S_{1}$ to which the Bethe wave function belongs. Hence the latter one turns out to be the ground state in $S_{1}$ but an excited one in $S_{2}$. The good agreement ensures that at least in one dimension the approximation introduced here is useful.

The IIubbard Hamiltonian has been diagonalised approximately in a pairwise basis. Within this Hilbert space, the electron gas splits into subsets of electron pairs, characterised by a common value of the centre of mass momentum. The number of pairs in each subset is invariant, so that the subsets are decoupled. Two kinds of many-body eigenstates are found: correlated and uncorrelated. The correlated states turn out to be of the BCS type whereas the uncorrelated pairs build up a Fermi gas of independent electrons distributed in two one-electron bands. The energy of the uncorrelated solution at $T=0$ in one dimension is found to agree well with the Bethe ansatz result for arbitrary electron concentration and Hubbard coupling.

I dedicate this work to the memory of my mother Jochweta and my niece Denise Lévy who died shortly before its completion. I am delighted to express my deep thankfulness to my wife and children, Rachel, Jérémie, Judith and my colleagues, P. Lederer, M. Oehlers and J.L. Raimbault, for providing constant encouragement during this quest for a simple picture of the many-body problem.

\section{References}

[1] D.C. Mattis, The Theory of Magnetism I, Springer Verlag, Berlin 1965.

[2] B.K. Chakraverty, M. Avignon, D. Feinberg, J. Less. Common Met. 150, 11 (1989).

[3] J. Bardeen, L.N. Cooper, J.R. Schrieffer, Phys. Rev. 108, 1175 (1957).

[4] E.H. Lieb, F.Y. Wu, Phys. Rev. Lett. 20, 1445 (1968).

[5] M.W. Long, Int. J. Mod. Phys. B 5, 865 (1991).

[6] M. Lavagna, Int. J. Mod. Phys. B 5, 885 (1991).

[7] N.W. Ashcroft, N.D. Mermin, Solid State Physics, HRW, International Editions, Philadelphia 1974.

[8] L.D. Landau, E.M. Lifshitz, Statistical Physics, Pergamon Press, London 1979.

[9] F.D.M. Haldane, J. Phys. C 14, 2585 (1981).

[10] J.R. Schrieffer, Theory of Superconductivity, W.A. Benjamin, New York 1967.

[11] P. Nozières, S. Schmitt-Rink, J. Low Temp. Phys. 59, 195 (1985).

[12] H. Shiba, Phys. Rev. B 6, 930 (1972). 